Arq. Bras. Med. Vet. Zootec., v.70, n.3, p.931-938, 2018

\title{
Desempenho de tambaquis (Colossoma macropomum) submetidos a restrição alimentar e a realimentação em tanques-rede
}

[Performance of tambaqui submitted feed deprivation and refeeding in cages]

\author{
E.L. Santos, A.C.L. Soares, O.L.D. Tenório, E.C. Soares, T.J. Silva, \\ L.F. Gusmão Júnior, E.L. Santos
}

Universidade Federal de Alagoas - UFAL- Centro de Ciências Agrárias - Rio Largo, AL

\section{RESUMO}

Objetivou-se avaliar o desempenho de tambaquis (Colossoma macropomum) submetidos à restrição alimentar e à realimentação em tanques-rede. Durante 60 dias, foram alocados 100 tambaquis (peso inicial de 8,0 \pm 0,5g), em 20 tanques-rede. Foram realizados quatro tratamentos: T1=animais alimentados diariamente (controle), T2=alimentados durante seis dias com um dia de restrição de ração $(6 \mathrm{~A} / 1 \mathrm{R})$, $\mathrm{T} 3=$ alimentados durante cinco dias com dois dias de restrição $(5 \mathrm{~A} / 2 \mathrm{R})$ e $\mathrm{T} 4$ = alimentados durante quatro dias com três dias de restrição (4A/3R). Os animais foram alimentados duas vezes ao dia, às oito e às 16 horas, até a aparente saciedade, com ração extrusada comercial $(42 \% \mathrm{~PB})$. Utilizaram-se cinco repetições para cada tratamento e cinco peixes por unidade experimental, em um delineamento inteiramente ao acaso. A coleta de dados para avaliação do desempenho produtivo foi feita com base no peso final, no ganho de peso, no consumo médio de ração, na conversão alimentar, no comprimento total, no comprimento padrão, na altura, no índice de cabeça e de perfil, na taxa de eficiência proteica, na taxa de crescimento específico e na sobrevivência. Os resultados foram submetidos à ANOVA e ao teste de Tukey $(\mathrm{P} \geq 5)$. $\mathrm{O}$ tratamento $5 \mathrm{~A} / 2 \mathrm{R}$ apresentou-se como a melhor ferramenta na redução de custos de produção sem prejuízo ao desempenho produtivo de juvenis de tambaqui.

Palavras-chave: aquicultura, crescimento compensatório, peixe nativo, manejo alimentar

\begin{abstract}
The aim of this research was to evaluate the performance of tambaqui juveniles (Colossoma macropomum) submitted to feed deprivation and refeeding in cages. For 60 days of experimentation, 100 tambaquis were used (initial weight $8.0 \pm 0.5 \mathrm{~g}$ ), distributed in 20 cages. The treatments were: T1=daily fed animals (control), $T 2=$ fed for six days and one day of restriction $(6 F / 1 R), T 3=$ fed for five days and two days of restriction $(5 F / 2 R)$ and $T 4=$ fed for four days with three days of restriction (4F/3R). With five replicates each, and five fish for each experimental unit, arranged in a completely randomized design. In accordance with treatments, the fishes were fed twice a day at 08:00am and 4:00pm. The data collection for the performance evaluation was based on final weight, weight gain, average feed intake, alimentary conversion, total length, standard length, height, index head, index profile, protein efficiency rate, specific growth rate and survival at the end of the experimental period. Results were submitted to ANOVA and Tukey test $(P \geq 5)$. Treatment $5 F / 2 R$ resulted in the best performance results, which is a tool in reducing production costs without loss in performance of tambaqui juvenile.
\end{abstract}

Keywords: aquaculture, compensatory growth, feed management, native fish

\section{INTRODUÇÃO}

A piscicultura é uma atividade zootécnica que vem desenvolvendo em um ritmo muito acelerado no Brasil. Esse índice é muito superior

Recebido em 13 de março de 2017

Aceito em 29 de junho de 2017

E-mail: elton@zootecnista.com.br ao obtido na maioria das atividades agropecuárias, mais tradicionais. Isso se deve ao fato de a piscicultura ter uma boa lucratividade e também às condições ambientais favoráveis, como clima tropical e alta disponibilidade de água (Oliveira, 2009). 
Desse modo, a tecnologia de criação de peixes em tanques-rede vem sendo amplamente difundida no Brasil, mostrando-se uma técnica promissora por propiciar a ocupação sustentável do meio ambiente com uma alta produtividade e densidade (Liranço et al., 2011).

O tambaqui (Colossoma macropomum) é uma das espécies nativas de peixes mais cultivadas no Brasil. Tal fato se dá principalmente em razão de sua rusticidade, de seu hábito alimentar onívoro e de seu crescimento rápido (Araújo-Lima e Gomes, 2010), além de fácil aceitação às rações artificiais e da adaptação à criação em cativeiro.

Dentro da cadeia produtiva da piscicultura intensiva, aproximadamente $70 \%$ do custo de produção são de gastos com a alimentação dos animais. Por isso, é de suma importância ter conhecimento não só da biologia como também do manejo alimentar dos peixes. Desse modo, estudos sobre o manejo alimentar ideal de ração para o máximo desenvolvimento do animal são essenciais para a sustentabilidade da atividade, de modo a se evitarem perdas e aumento nos custos com a produção.

O crescimento compensatório é uma estratégia de manejo alimentar bem documentada, usada para enfrentar o problema do alto custo de produção dos peixes, podendo ser empregado para melhorar sua taxa de crescimento e, ao mesmo tempo, diminuir os custos de produção (Santos et al., 2015).

Esse termo faz referência à habilidade do peixe para o desenvolvimento corpóreo mais rápido do que o normal após um período de jejum de alimento, o que o faz readquirir o seu peso original ou até aumentar a taxa de crescimento.

O crescimento compensatório, advindo de períodos curtos de privação alimentar e posterior realimentação em peixes, pode ter importante aplicação na aquicultura, visto que, além de otimizar a oferta e o consumo de ração pelos animais, também pode ser uma ferramenta de economia de custos com mão de obra humana para esse manejo.

Uma utilização acertada do fenômeno do crescimento compensatório após a privação alimentar pode resultar em maior produtividade, com aumento na taxa de crescimento e na eficiência alimentar e redução dos custos com alimentação (Wang et al., 2009).

Assim, o presente estudo tem como principal objetivo avaliar o efeito de quatro estratégias de manejo alimentar, que incluem períodos de restrição alimentar e realimentação, sobre o desempenho produtivo de tambaquis criados em tanques-rede.

\section{MATERIAL E MÉTODOS}

Durante um período de avaliação de desempenho de 60 dias, foram utilizados 100 juvenis de tambaqui (C. macropomum) com o peso médio inicial $8,0 \pm 0,5 \mathrm{~g}$. Os animais utilizados foram provenientes da Estação de Piscicultura de Itiúba, pertencente à Companhia do Desenvolvimento do Vale São Francisco (Codevasf, Brasil), no município de Porto Real do Colégio, Alagoas, Brasil.

O experimento foi conduzido em uma propriedade particular, localizada no município de União dos Palmares, Alagoas, Brasil (09 09' $46^{\prime \prime}$ de latitude sul, $36^{\circ} 01^{\prime} 55^{\prime \prime}$ de longitude oeste). Os peixes foram distribuídos em 20 tanques-rede de $0,5 \mathrm{~m}^{3}(0,5 \times 0,5 \times 0,5 \mathrm{~m})$ inseridos de forma linear a um viveiro escavado com dimensões de $2.700 \mathrm{~m}^{2}$ (45 x 60 x 1,5m), com renovação de água constante, a partir de uma nascente de água natural. Inicialmente foi realizado um período de sete dias de aclimatação, antes do período de avaliação experimental.

Os tratamentos constituíram na variação do manejo alimentar para os tambaquis, sendo utilizados quatro tratamentos (controle alimentação diária; 6A/1R - alimentação durante seis dias e privação de um dia; $5 \mathrm{~A} / 2 \mathrm{R}$ alimentação durante cinco dias e privação de dois dias; e 4A/3R - alimentação durante quatro dias e privação de três dias) e cinco repetições cada tratamento, dispostos num delineamento totalmente ao acaso. Cada tanque-rede com cinco peixe cada foi uma parcela experimental.

Em todos os tratamentos, foi utilizada ração comercial com $42 \%$ de proteína bruta (PB) em todos os tratamentos, indicada para peixes tropicais onívoros (Tab. 1), até a aparente saciedade. A ração foi ofertada duas vezes ao dia, de manhã e à tarde - às nove e às $15 \mathrm{~h}$, de acordo com cada tratamento. 
Tabela 1. Composição percentual da ração comercial $42 \%$ de PB

\begin{tabular}{ccc}
\hline Níveis de garantia & $(\%)$ & Ração comercial* \\
\hline Umidade & (Máx.) & $10,00 \%$ \\
Proteína bruta & (Mín.) & $42,00 \%$ \\
Extrato etéreo & (Mín.) & $10,00 \%$ \\
Matéria mineral & (Máx.) & $14,00 \%$ \\
Matéria fibrosa & (Máx.) & $4,00 \%$ \\
Cálcio & (Máx.) & $3,50 \%$ \\
Cálcio & (Mín.) & $2,80 \%$ \\
Fósforo & (Mín.) & $1,00 \%$ \\
\hline
\end{tabular}

*Níveis de garantia por $\mathrm{kg}$ do produto: vit. $\mathrm{A}=27.000,00 \mathrm{UI}$; vit. $\mathrm{D} 3=5.400,00 \mathrm{UI}$; vit. $\mathrm{E}=180,00 \mathrm{mg}$; vit. K3= 9,00mg; vit. $\mathrm{B} 1=9,00 \mathrm{mg}$; vit. $\mathrm{B} 2=18,00 \mathrm{mg}$; vit. $\mathrm{B} 6=18,00 \mathrm{mg}$; vit. $\mathrm{B} 12=18,00 \mathrm{mcg}$; vit. $\mathrm{C}=350,00 \mathrm{mg}$; ácido pantotênico $=72,00 \mathrm{mg} ;$ biotina $=0,90 \mathrm{mg} ;$ niacina $=270,00 \mathrm{mg} ; \mathrm{Fe}=18,00 \mathrm{mg} ; \mathrm{Cu}=72,00 \mathrm{mg} ; \mathrm{Mn}=225,0 \mathrm{mg} ; \mathrm{Zn}=$ $75,00 \mathrm{mg} ; \mathrm{I}=36,00 \mathrm{mg}$ e $\mathrm{Se}=0,90 \mathrm{mg}$.

Os indicadores de qualidade de água: $\mathrm{pH}$, oxigênio e temperatura foram monitorados diariamente, em dois horários (10h e $17 \mathrm{~h})$, por meio de sonda multiparamétrica da marca HANNA Instruments, modelo 9828, Woonsocket, USA, a uma profundidade de aproximadamente $10 \mathrm{~cm}$ abaixo da lâmina d'água. A amônia total $\left(\mathrm{NH}_{3}+\mathrm{NH}_{4}\right)$ foi mensurada, em avaliações a cada dois dias, com o auxílio de espectrofotômetro da marca HANNA Instruments, modelo HI 83203, Bélgica, utilizando-se os reagentes de modelo HI93700-01. O nitrito $\left(\mathrm{NO}_{2-}\right)$ foi mensurado com esse mesmo equipamento, utilizando-se os reagentes HI93707-01 para o nitrito.

Ao final dos 60 dias, todos os animais foram abatidos por choque térmico para serem submetidos à biometria. As variáveis avaliadas foram: peso final $(\mathrm{PF})$; ganho de peso $(\mathrm{GP}=$ peso final - peso inicial); comprimento total (CTOTAL), medido entre a parte inicial da cabeça até o final da nadadeira caudal; comprimento padrão (CPAD), compreendendo a medida anterior da cabeça até o início da nadadeira caudal; altura (ALTURA); índice de perfil (IPERF = comprimento padrão/altura); consumo médio de ração; conversão alimentar $(\mathrm{CA}=$ consumo de ração/ganho de peso total); índice de cabeça (ICAB = comprimento total/comprimento da cabeça); índice de perfil $(\mathrm{IP}=$ comprimento/altura $)$; taxa de sobrevivência $\left(\mathrm{SOBREV}=\mathrm{n}^{\mathrm{o}}\right.$ de peixes vivos $/ \mathrm{n}^{\circ}$ total de peixes $x$ 100); taxa de crescimento específico diário $(\mathrm{TCE}=[(\ln \mathrm{PF}-\ln \mathrm{PI}) / \mathrm{t}] \mathrm{x} 100)$ e taxa de eficiência proteica (T.E.P $=$ ganho de peso/consumo de proteína).

Todos os dados obtidos foram submetidos à análise de variância (ANOVA) e ao teste de comparação de médias de Tukey ao nível de 5\% de probabilidade.

Esta pesquisa está de acordo com os princípios éticos em pesquisa com animais e foi aprovada pelo Comitê de Ética no Uso de Animais da Universidade Federal de Alagoas, Brasil (Protocolo n ${ }^{\text {: }}$ 09 /2016 - Ceua/Ufal).

\section{RESULTADOS E DISCUSSÕES}

Durante o período experimental, foram observados os valores médios para a temperatura do viveiro escavado onde o experimento foi realizado, de $25,0 \pm 1,10^{\circ} \mathrm{C}$; para o oxigênio dissolvido, de 7,0 $\pm 1,08 \mathrm{mg} / \mathrm{L}$; para o $\mathrm{pH}$, de 6,8 $\pm 0,2$; para a amônia, de $0,10 \pm 0,15 \mathrm{ppm}$; para o nitrito, de $0,10 \pm 0,01 \mathrm{ppm}$; e para a dureza total, de $40 \mathrm{mg} / \mathrm{L}$. Durante todo o experimento, os valores permaneceram dentro da faixa considerada adequada para o desenvolvimento de tambaquis (Araújo-Lima e Gomes, 2010).

Os valores médios do desempenho produtivo dos animais, obtidos ao final do experimento, no presente estudo, estão apresentados na Tab. 2. 
Tabela 2. Valores médios de desempenho produtivo de juvenis de tambaqui submetidos a diferentes dias de restrição alimentar e realimentação

\begin{tabular}{ccccccc}
\hline Itens & Controle & $6 \mathrm{~A} / 1 \mathrm{R}$ & $5 \mathrm{~A} / 2 \mathrm{R}$ & $4 \mathrm{~A} / 3 \mathrm{R}$ & $\mathrm{CV}(\%)$ & Teste F \\
\hline PI $(\mathrm{g})$ & $8,00 \mathrm{a}$ & $7,92 \mathrm{a}$ & $8,80 \mathrm{a}$ & $8,94 \mathrm{a}$ & 12,87 & 1,19 \\
PF $(\mathrm{g})$ & $26,60 \mathrm{a}$ & $28,00 \mathrm{a}$ & $30,00 \mathrm{a}$ & $25,00 \mathrm{a}$ & 21,25 & 0,57 \\
GP $(\mathrm{g})$ & $18,89 \mathrm{a}$ & $20,40 \mathrm{a}$ & $21,00 \mathrm{a}$ & $16,40 \mathrm{a}$ & 25,38 & 0,88 \\
CMR $(\mathrm{g})$ & $60,00 \mathrm{a}$ & $48,00 \mathrm{~b}$ & $40,00 \mathrm{c}$ & $32,00 \mathrm{~d}$ & 0,00 & 1,00 \\
CA $(\mathrm{g})$ & $4,14 \mathrm{~b}$ & $2,97 \mathrm{ab}$ & $2,40 \mathrm{a}$ & $2,06 \mathrm{a}$ & 1,19 & 5,14 \\
CTOTAL $(\mathrm{cm})$ & $6,80 \mathrm{a}$ & $6,06 \mathrm{a}$ & $6,60 \mathrm{a}$ & $6,26 \mathrm{a}$ & 7,92 & 2,11 \\
CPAD $(\mathrm{cm})$ & $3,11 \mathrm{a}$ & $2,96 \mathrm{a}$ & $3,19 \mathrm{a}$ & $2,85 \mathrm{a}$ & 15,67 & 1,25 \\
ALTURA $(\mathrm{cm})$ & $2,66 \mathrm{a}$ & $2,62 \mathrm{a}$ & $2,74 \mathrm{a}$ & $2,74 \mathrm{a}$ & 15,19 & 0,09 \\
\hline
\end{tabular}

Letras diferentes na mesma linha expressam diferenças significativas pelo teste de Tukey a $5 \%$ de probabilidade. Peso inicial (PI), peso final (PF), ganho de peso (GP), consumo médio de ração (CRM), conversão alimentar (CA), comprimento total (CTOTAL) e comprimento padrão (CPAD).

Não foram verificadas diferenças $(\mathrm{P} \leq 0,05)$ para as variáveis peso final, ganho de peso, comprimento total, comprimento padrão e altura, entre as diferentes estratégias de manejo alimentar estudadas.

Entretanto, houve diferenças $(\mathrm{P} \leq 0,05)$ nas variáveis conversão alimentar e consumo médio de ração, sobrevindo uma diminuição constante do consumo médio de ração pelos animais à medida que se aumentava o período de privação alimentar. Isso pode ter ocorrido devido ao menor tempo de disposição de alimento aos peixes ou, também, à menor capacidade de ingestão de alimento advindo de menos dias de alimentação.

A conversão alimentar tem relação direta com a quantidade de alimento que o peixe ingere e a eficiência na sua transformação em tecidos corpóreos. Assim, como não houve diferenças no ganho de peso dos animais e o consumo foi menor à medida que se aumentava o período de privação alimentar, a conversão alimentar seguiu a mesma tendência direta de ser mais baixa, ou melhor, o que diferiu quando dos tratamentos com períodos de privação de dois ou três dias em relação ao tratamento controle. Contudo, o tratamento em que os peixes eram alimentados diariamente (controle) não diferiu $(\mathrm{P} \leq 0,05)$ do tratamento em que os animais foram privados de apenas um dia da oferta da ração (6A/1R).

A habilidade dos animais em recuperar o crescimento, quando realimentados após privação alimentar, é conhecida há muitos anos (Bilton e Robins, 1973). No entanto, Ali et al. (2003) destacam que períodos de jejum são naturais e comuns na vida dos peixes, seja devido a épocas em que há pouca disponibilidade de alimento, seja devido à diminuição na temperatura da água.

Resultados semelhantes foram encontrados por Kim e Lovell (1995), que não obtiveram diferenças no ganho de peso nem melhores resultados para a conversão alimentar em períodos curtos de restrição alimentar seguidos de realimentação, sendo isso, também, assim como no presente estudo, consequência do menor consumo de ração, quando trabalharam com o bagre-de-canal (Ictalurus punctatus) durante um período de avaliações desses ciclos de um e dois anos.

Os resultados encontrados no presente estudo para as variáveis morfométricas dos animais estão expressos na Tab. 3. 
Desempenho de tambaquis...

Tabela 3. Valores médios dos índices morfométricos de juvenis de tambaqui submetidos a diferentes dias de restrição alimentar e realimentação

\begin{tabular}{ccccccc}
\hline Itens & Controle & $6 \mathrm{~A} / 1 \mathrm{R}$ & $5 \mathrm{~A} / 2 \mathrm{R}$ & $4 \mathrm{~A} / 3 \mathrm{R}$ & $\mathrm{CV}(\%)$ & Teste F \\
\hline ICAB $(\mathrm{cm})$ & $4,00 \mathrm{ab}$ & $3,80 \mathrm{a}$ & $4,00 \mathrm{ab}$ & $4,10 \mathrm{~b}$ & 5,46 & 5,34 \\
IPERF $(\mathrm{cm})$ & $2,44 \mathrm{a}$ & $2,00 \mathrm{a}$ & $2,20 \mathrm{a}$ & $2,00 \mathrm{a}$ & 11,21 & 3,72 \\
TEP $(\% /$ dia $)$ & $0,83 \mathrm{ab}$ & $0,80 \mathrm{a}$ & $1,38 \mathrm{~b}$ & $1,25 \mathrm{ab}$ & 28,07 & 4,80 \\
TCE $(\% /$ dia) & $31,21 \mathrm{a}$ & $33,80 \mathrm{a}$ & $35,03 \mathrm{a}$ & $27,20 \mathrm{a}$ & 26,08 & 0,87 \\
SOBREV $(\%)$ & 100,00 & 100,00 & 100,00 & 100,00 & - & - \\
\hline
\end{tabular}

Letras diferentes na mesma linha expressam diferenças significativas pelo teste de Tukey a 5\% de probabilidade. Índice de cabeça (ICAB), índice de perfil (IPERF), altura (ALT), taxa de eficiência proteica (TEP), taxa de crescimento específico e sobrevivência (SOBREV).

Não foi observada nenhuma morte dos juvenis de tambaqui, registrando-se uma taxa de sobrevivência de $100 \%$ durante todo o período experimental, em todos os tratamentos. Também não foram verificadas diferenças $(\mathrm{P} \leq 0,05)$ para as variáveis morfométricas de índice de perfil e para os resultados de taxa de crescimento específico.

Entretanto, verificaram-se diferenças $(\mathrm{P} \leq 0,05)$ das diferentes estratégias de arraçoamento sobre a eficiência proteica (TEP) e o sobre o índice de cabeça (ICAB).

Os peixes alimentados durante cinco dias consecutivos, com dois dias de privação alimentar $(5 \mathrm{~A} / 2 \mathrm{R})$, apresentaram TEP $(\mathrm{P} \leq 0,05)$ maior do que aqueles peixes que estavam sob condições de seis dias de alimentação e um de restrição (6A/1R), mas não houve diferença $(\mathrm{P} \leq 0,05)$ em relação ao tratamento $4 \mathrm{~A} / 3 \mathrm{R}$.

Os maiores resultados para índice de cabeça foram observados nos peixes que passaram pelo maior tempo de privação alimentar $(4 \mathrm{~A} / 3 \mathrm{R})$, porém não se verificou diferença $(\mathrm{P} \leq 0,05)$ nos animais que foram privados da alimentação por dois $\operatorname{dias}(5 \mathrm{~A} / 2 \mathrm{R})$.

Os melhores resultados de ICAB foram para o tratamento combinado por seis dias de alimentação e um de restrição (6A/1R), todavia não foi observada diferença $(\mathrm{P} \leq 0,05)$ nos animais mantidos com alimentação diária (controle) nem naqueles mantidos com dois dias de restrição $(5 \mathrm{~A} / 2 \mathrm{R})$.

O maior tamanho da cabeça compromete o rendimento de filé; dessa forma, um maior valor para o ICAB não é desejável para a maioria das espécies de peixes de corte com valor comercial. Também é importante ressaltar que o ICAB difere em relação às espécies de peixes. Dessa forma, Reis Neto et al. (2012) relatam que o tambaqui possui uma proporção de cabeça em relação ao corpo do animal grande quando comparado a outros peixes.

Apesar disso, no presente estudo, por se tratar de animais ainda jovens, a possibilidade de desenvolvimento posterior das partes comestíveis dos animais ainda é grande, visto que a idade, o tamanho e a faixa de peso do animal influenciam diretamente no rendimento da carcaça e do filé (Basso et al., 2011).

Ituassú et al. (2004), quando trabalharam com juvenis de tambaqui submetidos a quatro períodos de privação (zero, 14, 21, 28 dias), observaram que, ao final do experimento, somente os peixes submetidos a uma condição alimentar de 14 dias de privação alcançaram a massa dos peixes alimentados sem privação. Esses mesmos autores ainda destacaram que quanto mais longos os períodos de privação alimentar, maior é o esforço metabólico corporal para esses peixes alcançarem um crescimento compensatório, após a realimentação, similar aos animais alimentados diariamente e com maior frequência. Tal fato evidencia que juvenis de tambaqui (C. macropomum) podem apresentar crescimento compensatório quando submetidos à privação alimentar seguida de realimentação em períodos curtos.

Igualmente, Santos et al. (2016), ao estudarem estratégias de manejo alimentar com restrição e realimentação sobre o desempenho de machos 
Betta splendens, concluíram que a estratégia de manejo alimentar de $6 \mathrm{~A} / 1 \mathrm{R}$, ou seja, seis dias de alimentação com um dia de restrição de alimento, pode ser aplicada como indicativo no cultivo, sem prejuízos ao desempenho do animal, o que corrobora os resultados encontrados na presente pesquisa, nos quais se observou que, quando foi utilizada a estratégia de seis dias de alimentação com um ou dois dias de restrição (6A/1R ou $5 \mathrm{R} / 2 \mathrm{R})$, os resultados não diferiram do que se observou no tratamento controle, ou seja, quando os animais não passaram por restrição alimentar, na maior parte dos parâmetros de desempenho e de índices morfométricos avaliados.

Soares et al. (2007), quando trabalharam com alevinos de tucunaré (Cichla monoculus), com manejo alimentar de um dia de restrição e seis dias de realimentação, também não verificaram diferenças na conversão alimentar, no ganho de peso e na taxa de crescimento específico (TCE) em relação aos indivíduos do tratamento controle, que eram alimentados diariamente.

Já Rosauer et al. (2009), ao trabalharem com alevinos de Sander vitreus, não encontraram resultados inferiores dos peixes que foram submetidos a protocolos de alimentação de cinco dias de alimentação e dois dias de restrição $(5 \mathrm{~A} / 2 \mathrm{R})$, obtendo total compensação no desempenho dos peixes quando comparados ao tratamento em que os animais foram alimentados todos os dias (controle).

Vale ressaltar ainda que vários fatores podem interferir no crescimento compensatório dos peixes após um período de privação alimentar, como a biologia da espécie (Ali et al., 2003; Ye et al., 2016), o sexo (Vainikka et al., 2012) e a temperatura da água, que é uma das principais variáveis externas capaz de influenciar o desenvolvimento dos peixes, inclusive quando se trata do manejo alimentar com restrição de alimento (Moreira et al., 2008). Assim, Souza et al. (2003) demonstraram que o crescimento de pacus (Piaractus mesopotamicus) submetidos a seis semanas de restrição e sete semanas de realimentação não é prejudicial, sendo esse tratamento indicado somente para outono e inverno, pois nessas condições esse manejo promove maior crescimento, menor custo com ração, maior conversão alimentar e maior receita líquida parcial, devido à diminuição do metabolismo dos peixes pelo fato de a temperatura da água ser menor.

Sealey et al. (1998) também observaram, para bagre-do-canal, uma melhor eficiência da produção mediante regimes alimentares que incluem restrição nos meses mais frios do ano, pois, nessa época, com a queda da temperatura, $o$ metabolismo, a ingestão de alimento, a digestão e a resposta imune dos peixes diminuem.

A composição da dieta é um outro fator que pode influenciar a resposta do animal a um período de privação alimentar, sendo o nível e a qualidade da proteína de grande importância para manter a imunidade alta dos peixes $\mathrm{e}$ fornecer os aminoácidos essenciais de que eles precisam para deposição de músculos e tecidos (Ye et al., 2016) . Assim, os resultados apresentados por Santos et al. (2010) constataram que juvenis de tambaqui subordinados à privação alimentar seguida de realimentação obtiveram melhor desempenho, melhor composição de carcaça e maior duração da hiperfagia quando alimentados com $36 \%$ de proteína bruta na ração e sem alteração nos níveis de exigência proteica. Destaca-se que, no presente estudo, a composição da ração foi a mesma para todos os tratamentos, com níveis nutricionais adequados à espécie.

Deficiências originadas de logos períodos de restrição de alimento em estágios de desenvolvimento podem comprometer a habilidade dos organismos de se recuperarem quando os níveis adequados de alimentação são estabelecidos.

Abdel-Hackim et al. (2009) estudaram o desempenho de híbridos de juvenis de tilápia criados e constataram um crescimento compensatório satisfatório, com redução de custos de alimentação em regime de privação alimentar moderada (um ou dois dias). No entanto, em caso de maior regime alimentar (três dias), eles não conseguiram expressar um bom desempenho quanto ao crescimento, para compensar o atraso de desenvolvimento devido à alimentação restrita, o que ratifica os resultados obtidos com tambaquis no presente estudo. Já Palma et al. (2010), ao avaliarem o manejo alimentar de juvenis da tilápia-do-nilo (Oreochromis niloticus), sugeriram a estratégia $5 \mathrm{~A} / 2 \mathrm{R}$, corroborando os resultados encontrados no presente estudo com juvenis de tambaqui. 
A depender da espécie do peixe e do tempo de exposição à restrição alimentar, o crescimento compensatório obtido pelo peixe pode não ser suficiente para alcançar a recuperação metabólica necessária em termos de desenvolvimento corpóreo em relação à situação em que o animal tivesse disponível alimentos diariamente.

Também a quantidade de alimento natural disponível no ambiente de cultivo pode ser crucial para que o crescimento compensatório possa expressar-se de forma plena sobre o desempenho dos animais, visto que, nos dias de privação de oferta de ração, os animais poderiam filtrar melhor a água e obter os nutrientes necessários para suprir em parte a sua dieta diária.

O tambaqui é um peixe onívoro, mas possui grande capacidade filtradora, o que pode ter contribuído para o desempenho satisfatório no presente estudo quando da utilização de estratégias de restrição alimentar. Mesmo os animais estando alojados em tanques-rede, a baixa densidade utilizada torna disponível uma grande quantidade de plâncton proveniente do viveiro escavado onde estavam acoplados esses tanques.

De forma geral, nas condições estudadas para juvenis de tambaqui (Colossoma macropomum), recomenda-se a estratégia com dois dias de restrição alimentar e cinco dias de realimentação como ferramenta de redução na quantidade de ração ofertada, no desperdício dela e, consequentemente, de diminuição nos custos de produção, sem afetar irreversivelmente e significativamente o desempenho dos animais. Tal estratégia pode ser utilizada em outros segmentos da cadeia produtiva, como destacado por Urbinati e Carvalho (2005) ao estudarem matrinxãs (Brycon cephalus). Esses autores recomendam a adoção de períodos curtos de restrição e realimentação como condições específicas de manejo sem causar prejuízos irreversíveis aos peixes e com redução nos custos de produção.

\section{CONCLUSÃO}

O tambaqui exibe crescimento compensatório quando submetido à privação alimentar; dessa forma, recomenda-se a estratégia de dois dias de restrição com cinco dias de realimentação para juvenis de tambaqui (Colossoma macropomum). No entanto, estudos com avaliações econômicas dessas estratégias devem ser realizados.

\section{REFERÊNCIAS}

ABDEL-HAKIM, N.F.; ABO STATE, H.A.; AL-AZAB, A.A.; EL-KHOLY, K.F. Effect of feeding regimes on growth performance of juvenile hybrid tilapia (Oreochromis niloticus x Oreochromis aureus). World J. Agric. Sci., v.5, p.49-54, 2009.

ALI, M.A.; NICIEZA, A.G.; WOOTTON, R.J. Compensatory growth in fishes: a response to growth depression. Fish Fish., v.4, p.147-190, 2003.

ARAÚJO-LIMA， C.A.R.M.; GOMES, L.C. Tambaqui (Colossoma macropomum). In: BALDISSEROTTO, B., GOMES, L.C. Espécies nativas para piscicultura no Brasil. Santa Maria: UFSM, 2010. p.175-204.

BASSO, L.; FERREIRA, M.W.; SILVA, A.R. Efeito do peso ao abate nos rendimentos dos processamentos do pacu (Piaractus mesopotamicus). Arq. Bras. Med. Vet. Zootec., v.63, p.1260-1262, 2011.

BILTON, H.T.; ROBINS, G.L. The Effects of starvation and subsequent feeding on survival and growth of fulton channel sockeye salmon fry (Oncorhynchus nerka). J. Fish. Res. Board Can., v.30, p.1-5, 1973.

ITUASSÚ, R.D.; SANTOS, G.R.S.; ROUBACH, R. et al. Desenvolvimento de tambaqui (Colossoma macropomum) submetido a períodos de privação alimentar. Pesqui. Agropec. Bras., v.3, p.1199-1203, 2004.

KIM, M.K.; LOVELL, R.T. Effect of overwinter feeding regimen on body weight, body composition and resistance to Edwardsiella ictaluri in channel catfish, Ictalurus punctatus. Aquaculture, v.134, p.237-246, 1995.

LIRANÇO, A.D.S.; ROMAGOSA, E.; SCORVO-FILHO, J.D. Desempenho produtivo de Pseudoplatystoma corruscans estocados em sistemas de criação: semi-intensivo (viveiro escavado) e intensivo (tanque-rede). Cienc. Rural, v.41, p.524-530, 2011. 
MOREIRA, I.S.; PERES, H.; COUTO, A. et al. Temperature and dietary carbohydrate level effects on performance and metabolic utilization of diets in European sea bass (Dicentrarchus labrax) juveniles. Aquaculture, v.274, p.153-160, 2008.

OLIVEIRA, R.C. O panorama da aquicultura no Brasil: a prática com foco na sustentabilidade. Rev. Intertox Risco Ambien. Soc., v.2, p.71-89, 2009.

PALMA, E.H.; TAKAHASHI, L.S.; DIAS, L.T.S. et al. Estratégias de alimentar com ciclos de restrição e realimentação no desempenho produtivo de juvenis de tilápia do Nilo da linhagem GIFT. Cienc. Rural, v.4, p.421-426, 2010 .

REIS NETO, R.V.; FREITAS, R.T.F.; SERAFINI, M.A. et al. Interrelationships between morphometric variables and rounded fish body yields evaluated by path analysis. Rev. Bras. Zootec., v.41, p.1576-1582, 2012.

ROSAUER, D.R.; MORRIS, J.E.; CLAYTON, R.D. Role of compensatory growth in walleye fingerling production. N. Am. J. Aquac., v.71, p.35-38, 2009.

SANTOS, E.L.; SOARES, E.C.; SILVA, T.J. et al. Restrição alimentar no desempenho de machos do peixe beta (Betta splendens). Comunic. Sci., v.7, p.12-23, 2016.

SANTOS, E.L.; SOUZA, A.P.L.; PONTES, E.C. et al. Folha de amendoeira (Terminalia catappa) como aditivo promotor de crescimento em rações para alevinos de Tilápia do Nilo (Oreochromis niloticus). Agropecu. Tec., v.36, p.190-196, 2015.

SANTOS, L.; PEREIRA FILHO, M.; SOBREIRA, C. et al. Exigência proteica de juvenis de tambaqui (Colossoma macropomum) após privação alimentar. Acta Amazonica, v.40, p.597-604, 2010.
SEALEY, W.M.; DAVIS, J.T.; GATLIN III, D.M. Restricted feeding regimes increase production efficiency in channel catfish. Auburn: SRAC, 1998. 6p. (Publication n.189).

SOARES, C.E.; PEREIRA FILHO, M.; ROUBACH, R. et al. Condicionamento alimentar no desempenho zootécnico do tucunaré. Rev. Bras. Eng. Pesca, v.2, p.35-48, 2007.

SOUZA, L.V.; URBINATI, E.C.; MARTINS, M.I.E.G. et al. Avaliação do crescimento e do custo da alimentação do pacu (Piaractus mesopotamicus) submetido a ciclos alternados de restrição alimentar e realimentação. Rev. Bras. Zootec., v.32, p.19-28, 2003.

URBINATI, C.E.; CARVALHO, G.E. Crescimento, desenvolvimento gonadal e composição muscular de matrinxãs (Brycon cephalus) submetidos à restrição alimentar $\mathrm{e}$ realimentação durante um ano. Cienc. Rural, v.35, p.897-902, 2005.

VAINIKKA, A.; HUUSKO, R.; HYVÄRINEN, P. et al. Food restriction prior to release reduces precocious maturity and improves migration tendency of Atlantic salmon (Salmo salar) smolts. Can. J. Fish. Aquac. Sci., v.69, p.19811993, 2012.

WANG, Y.; LI, C.; QIN, J.C. et al. Cyclical feed deprivation and refeeding fails to enchance compensatory growth in Nile tilapia, Oreochromis niloticus L. Aquacult. Res., v.40, p.204-210, 2009.

YE，J.D.; CHEN， J.C.; WANG，K. Growth performance and body composition in response to dietary protein and lipid levels in Nile tilapia (Oreochromis niloticus Linnaeus, 1758) subjected to normal and temporally restricted feeding regimes. J. Appl. Ichthyol., v.32, p.332338, 2016. 